\title{
Perspective and Experience of Hospital Operating Room Nurses with the Concept of Excellence: A Qualitative Study
}

This article was published in the following Dove Press journal: Risk Management and Healthcare Policy

\section{Soheila Bakhtiari ${ }^{1,2}$ \\ Farkhondeh Sharif ${ }^{3}$ \\ Mohsen Shahriari ${ }^{4}$ \\ Mahnaz Rakhshan ${ }^{5}$}

'Student Research Committee, Shiraz University of Medical Sciences, Shiraz, Iran; ${ }^{2}$ Operating Room Department, School of Nursing and Midwifery, Nursing and Midwifery Care Research Center, Isfahan University of Medical Sciences, Isfahan, Iran; ${ }^{3}$ Community Based Psychiatric Care Research Center, School of Nursing and Midwifery, Shiraz University of Medical Sciences, Shiraz, Iran; ${ }^{4}$ Department of Adult Health Nursing, School of Nursing and Midwifery, Nursing and Midwifery Care Research Center, Isfahan University of Medical Sciences, Isfahan, Iran;

${ }^{5}$ Community Based Psychiatric Care Research Center, School of Nursing and Midwifery, Shiraz University of Medical Sciences, Shiraz, Iran
Correspondence: Mahnaz Rakhshan Community Based Psychiatric Care Research Center, School of Nursing and Midwifery, Shiraz University of Medical Sciences, Shiraz, Iran

Emailmahnazrakhshan@yahoo.com
Introduction: The provision of care according to the code of ethics is of the highest priority in operating rooms (OR). However, the exposure of the surgical team to a high level of stress may result in unethical behavior and undermine their pursuit of excellence. Since the concept of excellence is complex and there are limited published studies in the nursing literature, there was a need for in-depth research.

Objective: The present study aimed at evaluating the perspective and experience of OR nurses with the concept of excellence.

Study Design: The conventional qualitative content analysis method was employed to explore the concept of excellence among OR nurses.

Participants and Research Environment: The current study was conducted on 20 OR nurses in the elective and emergency operating rooms of hospitals affiliated to Isfahan University of Medical Sciences, Isfahan, Iran. The data were collected through in-depth semi-structured face-to-face interviews and field notes from April 2017 to June 2018.

Findings: The four categories extracted from the interview data were "enhanced personality traits", "growth and development", "knowledge enhancement", and "effective teamwork". In addition, a total of 10 sub-categories were extracted.

Discussion: The findings of the present study indicated that OR nurses can achieve personal, professional, organizational, and social excellence through enhancing personality traits, peer learning, teaching ethics to the surgical team members, and educating and training patients and family caregivers through effective teamwork. A close collaboration between OR nurses and nursing managers would develop a culture of pursuit for excellence.

Conclusion: The findings of the present study provided a better understanding of the perception of OR nurses with the concept of excellence; based on which, optimal ethical care and an environment for the pursuit of excellence can be developed. The findings also provided evidencebased recommendations to nursing managers on how to gain the trust of patients and family caregivers, and promote the pursuit of personal, professional, organizational, and social excellence. Keywords: excellence, professional values, nursing, operating room, positive psychology, content analysis, qualitative research

\section{Introduction}

Plato asserts that "virtue" is the sole human trait that ensures the prosperity of both individuals and society through a harmonious combination of knowledge, power, and desire. According to Socrates, excellence is constituted by ethical virtue, justice, beauty, and benefit. ${ }^{1}$ The overall perception of excellence is formed based on ontological, epistemological, anthropological, aesthetic, and methodological components. ${ }^{2}$ Aristotle 
and Plato assert that individuals achieving excellence have a clear understanding of their role in the universe, have respect for their environment, pursue knowledge, and love all that surrounds them. Such people are pure, noble, and ambitious; live a harmonious life, fulfill social duties, and actively participate in social affairs. ${ }^{3}$

The pursuit of excellence is a continuous process and fundamental to achieving professional goals. It encompasses striving for the highest standard, consistency, continuity, and providing high quality and reliable services. ${ }^{4}$ Previous studies on the concept of excellence mainly focused on the quality of organizations and management. Some of these studies employed organizational excellence models, of which the European Foundation for Quality Management (EFQM) is the most renowned. ${ }^{5-7}$ A study at the University of Kentucky (USA) on the concept of excellence among hospital managers and nursing employed the appreciative inquiry (AI) model. ${ }^{8}$ Based on the theory of Corbin and Strauss, a study in Iran developed and introduced a functional model to assess the professional excellence of nurses. ${ }^{9}$

Nowadays, unethical behavior poses a serious challenge to healthcare providers, national economies, and societies. ${ }^{10,11}$ Such behaviors are common in the workplace; however, many organizations falsely tend to ignore the issue in the strive to remain commercially competitive and enlarge their market share. ${ }^{12}$ Hospital operating rooms (OR) are not exempted from such tendencies since they are the most profitable section in a hospital. ${ }^{13-16}$ Although excessive pressure and the uniqueness of working in the OR may result in unethical behaviors, the general public is, however, very sensitive to such behaviors as they have a direct impact on the health and well-being of patients. ${ }^{17-19}$ Regrettably, although preventable, most of the reported unethical behaviors in health care take place in the $\mathrm{OR}^{20}$ Since activities in the OR are highly technical, consequently, the provision of care is negatively affected by timeconsuming technical procedures. ${ }^{21}$ Moreover, the surgical team is constantly under pressure to meticulously perform a complex surgery and patient's care is provided by multiprofessional and interprofessional teams. ${ }^{22,23}$ The exposure of the multi-disciplinary surgical team to a high level of stress in a high-risk environment may result in susceptibility to ethical distress and unethical behavior. ${ }^{24-26}$

Noting the above-mentioned assertion by Socrates, one can also deduce a direct association between the nursing profession and the pursuit of excellence, since values such as ethical virtue, justice, beauty, and benefit are also part of the nursing code of ethics upon which nurses act and make decisions. Unfortunately, according to the authors, the negative impact of technological advancement, globalism, and materialism force the nursing profession to distance itself from its ethical values. Since the concept of excellence is complex and there are limited published studies in the nursing literature, there was a need for in-depth research. Authors anticipate that the mere participation of OR nurses in the present study generated awareness and would contribute toward professional excellence in nursing.

\section{Objective}

The present study aimed at identifying the perspective and experience of OR nurses with the concept of excellence.

\section{Research Design}

The conventional qualitative content analysis method was employed to explore the concept of excellence among OR nurses.

\section{Participants and Research Environment}

The present study was conducted on OR nurses in the elective and emergency operating rooms of hospitals affiliated to Isfahan University of Medical Sciences, Isfahan, Iran. The purposive sampling method was employed to recruit the subjects. The inclusion criteria were a minimum of sixmonth work experience, holding at least a bachelor's degree, mental and psychological ability to participate in an interview, and willingness to participate.

\section{Data Collection}

The data were collected through in-depth semi-structured face-to-face interviews from April 2017 to June 2018. The sampling process was continued until data saturation was reached. ie, until no new categories emerged and the obtained categories were saturated in terms of features and dimensions. The location of the interview was chosen jointly by the participants and each interview (conducted by the first author) lasted approximately 50 mins. Prior to the interviews, all participants were informed about the study objectives and procedures. The confidentiality of any disclosed information was guaranteed and written informed consent was obtained from all the participants.

The interviews started with a general question: "What is your understanding of the term excellence?" Then followed by a series of detailed questions such as "What are your personal experiences with achieving excellence in the 
OR?" and "Which actions are taken by the surgical team toward achieving excellence?" With the permission of the participants, an audio recording of the interviews was made and, subsequently, transcribed verbatim, reviewed, and coded. In addition, field notes were taken during the morning and evening shifts in the operating room.

\section{Data Analysis}

The data were analyzed using conventional content analysis (Lundman and Graneheim, 2004) and the constant comparative method. ${ }^{27}$ At the end of each interview, the audio recording together with the field notes were meticulously transcribed. Following multiple reviews of the transcription, initial semantic units (primary codes) were identified. The extracted codes were then classified in terms of similarities and differences based on which the sub-categories were defined. Then, they were reviewed, compared, and grouped to determine the general categories. Data analysis was performed using MAXQDA 10 software.

\section{Rigor}

The criteria proposed by Guba and Lincoln were used to confirm the validity of the study. ${ }^{28}$ The OR nurses and technologists were observed during different morning and afternoon shifts and in different ORs (triangulation in time and space). In addition, a sample of the interview transcriptions and primary codes were presented to the participants. Then, with their cooperation, the degree of homogeneity of the extracted categories was reviewed and confirmed by the research team. To achieve proportionality, a sampling technique with a maximum variation (age, gender, and work experience) was used. To fulfill the transferability criterion, a full description of the results, the data analysis, and quotations of the participants' statements were provided. The credibility and dependability criteria were confirmed through extensive teamwork by the research team members combined with interaction and supervision of experts.

\section{Ethical Considerations}

The present study was approved by the Ethics Committee of Shiraz University of Medical Sciences, Shiraz, Iran (ethical code: IR.SUMS.REC.1396.S600). The location of the interview was chosen jointly by the participants. Prior to the interviews, the study objectives and methods were explained to all participants; in addition, they were assured of the confidentiality of any disclosed information and were asked to sign the informed consent form].

\section{Results}

The study was conducted on a total of 20 OR nurses. The majority of the participants were female $(n=15)$. The mean age and years of working experience were $40.6 \pm 9.06$ and $15.95 \pm 7.78$ years, respectively. The demographic characteristics of the participants are presented in Table 1.

The four categories extracted from the collected data were "enhanced personality traits", "growth and development", "knowledge enhancement", and "effective teamwork". A total of 10 sub-categories were also extracted (Table 2).

\section{Enhanced Personality Traits}

Improving character traits was a category of the concept of excellence that included sub-categories such as "improve moral courage" and "put spirituality into practice." The participants regarded the moral courage to openly express professional views in the OR as an important aspect of character traits. A participant stated:

We could not trace one sterile gauze during a surgery. I suspected that the gauze had been left behind in the patient's abdomen, but the surgical resident was adamant and initiated the procedure to close the abdominal wall. I refused to provide suture threads pending X-ray examination of the abdomen to find the missing gauze. The result was negative, but I had a hunch that the gauze had been left behind in the abdomen. To the dismay of the

Table I Demographic Characteristics of the OR Nurses

\begin{tabular}{|l|l|l|l|}
\hline Participant & Age (yr) & Gender & Working Experience (yr) \\
\hline 1 & 39 & Male & 20 \\
2 & 27 & Female & 4 \\
3 & 29 & Male & 5 \\
4 & 41 & Female & 21 \\
5 & 26 & Female & 4 \\
6 & 44 & Female & 23 \\
7 & 26 & Female & 5 \\
8 & 36 & Female & 16 \\
9 & 39 & Female & 17 \\
10 & 28 & Female & 3 \\
11 & 51 & Female & 25 \\
12 & 40 & Female & 17 \\
13 & 49 & Female & 12 \\
14 & 51 & Female & 25 \\
15 & 49 & Male & 23 \\
16 & 43 & Female & 21 \\
17 & 49 & Female & 24 \\
18 & 50 & Male & 14 \\
19 & 47 & Male & 18 \\
20 & 48 & Female & 22 \\
\hline
\end{tabular}


Table 2 Extracted Categories and Sub-Categories from the Collected Data

\begin{tabular}{|l|l|l|}
\hline \multirow{y}{*}{ Excellence } & Category & Sub-Category \\
\cline { 2 - 3 } & $\begin{array}{l}\text { Enhanced } \\
\text { personality traits }\end{array}$ & $\begin{array}{l}\text { Improve moral courage } \\
\text { Put spirituality into practice }\end{array}$ \\
\cline { 2 - 4 } & $\begin{array}{l}\text { Growth and } \\
\text { development }\end{array}$ & $\begin{array}{l}\text { Knowledge transfer among surgical } \\
\text { team members } \\
\text { Teaching ethical standards to the } \\
\text { surgical team }\end{array}$ \\
\cline { 2 - 3 } & Knowledge & $\begin{array}{l}\text { Educate and train the patients } \\
\text { Educate and train family caregivers }\end{array}$ \\
\cline { 2 - 3 } & Effective teamwork & $\begin{array}{l}\text { Collaboration among the surgical team } \\
\text { Effective learning and teaching process } \\
\text { Oversee the performance of team } \\
\text { members } \\
\text { Information exchange among team } \\
\text { members }\end{array}$ \\
\hline
\end{tabular}

surgical resident, I asked the main surgeon to attend. Luckily, he found the missing gauze in the abdomen and the issue was resolved. My courage to take appropriate actions was acknowledged and viewed as the pursuit of excellence. (P13)

The majority of the participants regarded spirituality as an important personality trait that positively affects their behavior toward moral excellence. Putting spirituality into practice was described by a participant as:

One should always remember that there is a God and our actions will be appraised on Judgment Day. If you sincerely believe it, you will always try to do the right things. Spirituality lifts one's moral behavior. (P14)

\section{Growth and Development}

The participants regarded growth and development of the surgical team as another category of the pursuit of excellence. The sub-categories associated with growth and development were "knowledge transfer among the surgical team members" and "teaching ethical standards to the surgical team". The participants stated that knowledge transfer between colleagues has the benefit of technological empowerment of the OR personnel and creates a dynamic work environment. In this regard, a participant stated:

Knowledge transfer between team members encourages cooperation between disciplines and turns the OR into an academic environment. Ultimately, the patients, personnel, and the hospital will benefit from such pursuit of excellence. (P7)
Teaching ethical standards to the surgical team was also emphasized by the participants. A participant stated:

At the beginning of my nursing career, senior colleagues taught me a lot about their experience with ethical behavior. They advised me to respond to patients' misbehavior with calmness and politeness to prevent a cycle of unethical behavior. One aspect of self-improvement is to control your temper. (P3)

\section{Knowledge Enhancement}

The participants regarded enhancing the knowledge of both the patients and family caregivers as another element of the pursuit of excellence. The provision of targeted medical information and procedures encourages the patients to better cooperate with the surgical team and gains the support of the family caregivers. As a direct result and also in the long term, the community benefits from such promotion of knowledge and the awareness of medical issues. A participant stated:

Once a female patient was admitted for a cesarean section. As it was her first pregnancy, we provided information on the advantages and disadvantages of a cesarean section and types of anesthesia (general or local). We highlighted that spinal anesthesia will allow her to be awake during labor and she could see her child immediately after birth. The patient was immensely appreciative. (P18)

\section{Effective Teamwork}

Teamwork was another category associated with the excellence of OR nurses. It included the sub-categories "collaboration among the surgical team members", "oversee the performance of team members", "effective learning and teaching process", and "information exchange among team members".

The participants regarded collaboration within the surgical team as the key element of successful teamwork. A participant stated:

We had a case of an unexpected cardiac arrest during surgery. The anesthesia technologist noticed a defective IV line for the administration of drugs. He panicked as he failed a new venipuncture attempt, while at the same time, he had to leave the OR to obtain drugs. Beyond my responsibility as the circulating nurse, I intervened and asked him to obtain the medicine, while I attempted venipuncture. Fortunately, it all worked out well and together we managed to stabilize the patient. Effective teamwork allowed us to provide an excellent service to the patient. (P19) 
The sub-category "effective learning and teaching process" was also viewed as an important element of successful teamwork. The combination of learning from and teaching each other was directly associated with effective teamwork.

The participants stated mixed experiences in overseeing the performance of team members. Some viewed that overseeing the performance of higher-ranking team members (eg surgical resident) was also a key element of successful teamwork. Whereas some others were culturally reluctant to question higher-ranking team members. There was an overall agreement on the need for dedicated training on the role of organizational hierarchy to overcome such restrictive cultural tendencies.

Timely information exchange among team members was regarded as essential to successful teamwork as it can prevent medical errors. A participant stated:

I took a patient to the OR and as the anesthesiologist was ready to administer the drug, I informed him that the patient was pregnant. He could not believe what he had heard and cross-checked it with the patient. The surgeon was also surprised and together they decided to cancel the surgery. My timely exchange of information proved its importance as it was crucial at that point of time. (P10)

There was a general agreement among the participants that the OR team requires training on effective teamwork. A participant stated:

We all can learn from each other, while we work together in the OR. I have learned a lot from my team members and in return, I will do my utmost to teach all I know to my direct colleagues. By doing so, together we will achieve optimum results. (P2)

\section{Discussion}

The present qualitative study aimed at identifying the perspective and experience of OR nurses with the concept of excellence. The analysis of the interview data resulted in four main categories, namely "enhanced personality traits", "growth and development", "knowledge enhancement", and "effective teamwork."

At first glance, in a challenging environment such as the OR, the pursuit of excellence should be the least of the worries of the surgical team. However, through positive thinking, individuals still strive for excellence even under the most extreme circumstances. ${ }^{29}$ The current study findings indicated that even in the OR, being the most challenging unit in a hospital, the surgical team still pursues personal, professional, and organizational excellence.

\section{Enhanced Personality Traits}

The results showed that the enhancement of personality traits was an important aspect of the concept of excellence. However, the challenges associated with a closed and stressful environment such as the OR (eg complex instruments, unpleasant scenes, patient life or death situation, fatigue and exhaustion, distress, unethical encounters, and interaction between different individuals and personalities) can negatively affect OR personnel and alter their behavior over time. Hence, the surgical team should continuously strive to enhance their moral character through selfreflection and self-improvement.

Seemingly, the presence of a suitable atmosphere is a requisite for humans to improve their skills. However, a new approach in psychology, called "positive psychology", explores the ability of people to focus on achieving success rather than the shortcomings of the surrounding conditions. ${ }^{30}$ Positive psychology is a mental approach to achieve the best possible outcome from the worst possible situation. A person with a positive mindset does not heed to life's shortcomings, but instead fights back to correct inadequacies. ${ }^{31}$ Positive psychology aims at identifying and enhancing those characteristics that enable an individual and the society to develop and grow. ${ }^{29}$ Hence, it is also possible to aim for excellence in a high-risk and stressful environment such as the OR. Farabi, a great Iranian philosopher, considered combining theory and practice to achieve excellence. He believed that individuals would achieve excellence if they adhere to what is considered to be good and dignified conduct, and by putting this into practice through good deeds and good behavior. ${ }^{32}$

The participants noted a range of personality traits (eg courage and spirituality) that facilitated personal and professional excellence. A study reported that the display of professional behavior beyond the work setting was a category of excellence in nursing practice and one of its sub-categories was the enhancement of personality traits. ${ }^{33}$ Whereas the current study found enhancing personality traits to be the main category, indicating its importance among OR nurses in pursuit of excellence. The participants viewed moral courage as the most important personality trait, which therefore required primary focus. They believed that the safety and well-being of a patient should not be undermined by the power play between the surgeons and other team members. Moral courage is an act based on ethical values upheld during difficult ethical dilemmas, despite adversity and even personal risk. $^{34,35}$ 
Most of the participants believed that moral courage is the primary trait that requires strengthening. They stated that in the power struggle with surgeons, OR nurses should not sacrifice the interests of patients by being submissive. Ethical courage means having the bravery to act based on ethical values despite the difficulties and vulnerability posed by ethical challenges and dilemmas. ${ }^{34,35}$ Moral courage is an ethical value that enables nurses to overcome their emotions instinct, and the fear of reprisal in order to consciously and resolutely take appropriate action against unethical behaviors. ${ }^{36,37}$ Organizational ethics and values can directly influence the moral courage of personnel. ${ }^{38}$ As a result, the personnel of organizations where ethics are highly valued tend to attain a higher level of moral courage over time. Health systems and healthcare organizations consider an appropriate level of moral courage among their personnel as an asset, since it enhances professional ethical behavior ${ }^{39}$ and drives the organization toward professional and organizational excellence.

The majority of the participants emphasized the importance of putting spirituality into practice in the workplace. They trusted that belief in God and Judgment Day would prevent unethical behavior, particularly in the seclusion of the OR, where patients are unconscious during surgery and cannot respond. Moreover, a study indicated that spirituality is an important factor in strengthening resilience. ${ }^{40}$ The participants in the present study believed that spiritually empowered them to overcome difficulties in personal and professional dimensions of their lives. In the literature, spirituality is considered as having a relationship and belief in God as the supreme power and excellence. ${ }^{40}$ In most religions (Christianity and Islam), spirituality is directly linked with religion. ${ }^{41}$ However, spirituality encompasses a wider concept than religion, since even those who do not believe in God may have a sense of spirituality. ${ }^{42,43}$ According to Watson's theory, spirituality is the capacity of human beings to enable selfawareness and heightened consciousness, which provides the inner strength to transcend his/her usual self. ${ }^{44}$ Spirituality can result in regaining health, recovering from sufferings, increased adaptability, tranquility, hope, and personal excellence of individuals. ${ }^{45}$ It is believed that those who have reached personal excellence through spirituality can positively affect the health system and considerably contribute to its organizational excellence.

\section{Growth and Development}

The participants regarded growth and development of the surgical team as a category of the concept of excellence. The sub-categories associated with growth and development were "knowledge transfer among surgical team members" and "teaching ethical standards to the surgical team". The participants stated that both the ethical and technical knowledge exchanged among colleagues can result in personal and professional excellence and enhance the dynamics of the work environment in the OR. The current study findings indicated that competent OR personnel can enhance the knowledge and performance of their team members by sharing their knowledge. This, in turn, generates a dynamic atmosphere and a breeding ground for acquiring knowledge in the workplace. Clinical educators and nurses play an important role in the pursuit of knowledge. In line with the current study findings, a previous study classified knowledge transfer as a subcategory of professional excellence in the nursing profession. ${ }^{33}$ The findings also indicated that those who teach ethical practices to team members were committed to the essence of nursing and contributed to the next generation of professional ethical care providers. This is a key element of excellence, since such professionals can reform an organization in terms of its culture, management, operation, and the provision of optimal service. ${ }^{46}$ Another study used leadership skills to develop certain qualities among the participants and encouraged them to become a professional role model within the organization. ${ }^{33}$ The fact that the teaching of ethical standards was extracted as a sub-category of growth and development was indicative of its importance in the current study. The participants emphasized the importance of ethical standards for OR nursing and recruitment of individuals who behave ethically. The current study results showed that in the long-term, teaching ethical standards encourages others to behave ethically, which ultimately generates an ethical environment in the OR. Obviously, this cannot be a mere theoretical teaching process, but rather requires the actual practice of ethical principles as well.

\section{Knowledge Enhancement of Patients and Family Caregivers}

Teaching and training patients and family caregivers were the sub-categories of excellence. Even though patients are anesthetized upon arrival at the OR and their interaction with OR nurses is brief, there is still a need for verbal communication with such patients. The participants believed that it is their ethical and professional responsibility to provide adequate information whenever possible. Receiving appropriate information from the medical personnel is clearly stated in the Patients' Rights Charter. ${ }^{47}$ 
Although both physicians and nurses acknowledge such rights, regrettably it is not always put into practice. ${ }^{48}$ The findings of the present study indicated that the provision of information creates a sense of optimism and hope among patients and caregivers. In addition, it generates a positive picture of the nursing profession and elevates their status within the community. Confidence building measures combined with the provision of medical information put patients at ease and gives them a sense of being cared for. ${ }^{49}$ Clearly, the displeasure of patients and family caregivers with these aspects would cast doubt on the quality of nursing care as well as their ethical behavior. Overall, both the "growth and development" by the surgical team and the "teaching and training of the patients and family caregivers" are the contributing factors in pursuit of excellence by OR nurses, hospital managers, patients, family caregivers, and ultimately society as a whole.

\section{Effective Teamwork}

Teamwork was a category of excellence with four subcategories. The participants regarded effective collaboration within the surgical team as a contributing factor to improve self-image, self-confidence, and job satisfaction. In addition, coherent teamwork contributed to the provision of safe and excellent care, patients' satisfaction, and enhancement of the performance of the OR personnel. Various studies indicated that effective teamwork in the OR was directly associated with patients' safety, which of course is the main goal of all healthcare centers when providing optimal care and organizational excellence. ${ }^{50,51}$ Noting that several multidisciplinary teams are working together in the OR, any mistake or deviation from the assigned duties may have a huge effect on the patient's wellbeing. Therefore, effective teamwork in the OR is of paramount importance. ${ }^{52}$ Teamwork combines knowledge, skill, and attitude of the team members, and requires aspects such as communication, creative behavior, leadership, workload management, care process monitoring, and stress management. ${ }^{53,54}$ Nowadays, the complexity of care changes in the care environment, and the emergence of various fields of medical specialization negatively affect the collaboration between different medical professionals. Therefore, education and training programs to improve effective teamwork are essential. $^{49,55}$ Such programs could enhance the mindset of medical professionals, strengthen clinical care and teamwork processes, and even improve patients' outcomes. ${ }^{56}$

The majority of the participants regarded collaboration as a success factor in effective teamwork. However, collaboration should be in the form of productive support and communication rather than interference with others' expertise. Yet, collaboration within the surgical team is a challenging task, since the team includes different professionals (surgeon, anesthesiologist, and OR nurses) each with a specific mentality, culture, values, beliefs, attitudes, and behaviors. ${ }^{57}$ These professionals tend to focus on their own field of expertise, resulting in ineffective peer-to-peer communication and coordination, ${ }^{58}$ while the provision of quality care requires harmony, effective communication, and teamwork within the surgical team. ${ }^{59}$

The findings of the present study indicated that an effective learning and teaching process is an aspect of teamwork that led to personal, professional, and organizational excellence. Learning and teaching can take place within the dynamics of team collaboration and require an open educational environment. According to Koppman, learning is a requisite for the pursuit of excellence and implies a need for the improvement of the learning process. ${ }^{60}$ The current study findings also indicated the need for training the OR personnel on teaching and learning as part of an effective teamwork program. Although the OR personnel are experts in their field, they lack the necessary education and training on how to effectively act within a team. ${ }^{53}$ It is proposed that teamwork training should be in the form of interprofessional education, whereby the team members learn from, with, and about each other's skills. ${ }^{61,62}$

Overseeing the performance of the team members was also stated as an element of effective teamwork, as the main category of excellence. The surgical team bears the end responsibility for the well-being of patients, and consequently, each team member should not shy away from overseeing the performance of their direct colleagues. However, such supervision across the hierarchy is culturally not accepted in Iranian society and viewed as interference and intrusion. This implies the need to build an organizational culture emphasizing collaboration between nursing managers and personnel. Nonetheless, monitoring direct colleagues by providing training and coaching to those with less optimal performance is inherent to teamwork. $^{63}$ Based on the current study results, it was also determined that if the supervision is conducted properly, possible errors are identified and managed at an early stage, which is in line with the pursuit of excellence.

The current study findings indicated that information exchange among team members was essential toward effective teamwork; however, it requires a positive and open approach as well as strong communication skills. The OR personnel appeared to lack the necessary communication skills. Education and training on how to communicate effectively 
are needed, for which interprofessional training is recommended. Based on the participants' experiences, direct information exchange and verification of the given information ensures achieving optimal patients' outcome. However, it appears that a minimum standard of information exchange between the surgical team members is already established. As reported in a previous study, lack of control and verification of information in accordance with standard procedures could ultimately put patients' safety at risk and undermine excellence in nursing care. ${ }^{64}$

Overall, the findings of the present study indicated that OR nurses can achieve personal, professional, organizational, and social excellence through enhancing personality traits, peer learning, teaching ethical standards to the surgical team members, educating and training patients and family caregivers, and effective teamwork. The latter was regarded as an essential element in the pursuit of excellence, which requires dedicated planning and repetitive training sessions. In this process, close collaboration between nursing managers and OR nurses to develop a culture of striving for excellence is recommended.

\section{Limitations and Recommendations}

The main limitation of the present study was the participation of OR nurses from educational hospitals affiliated to one Iranian university of medical sciences, which undermined the generalizability of the findings. Future inclusion of physicians, as well as medical students, should give a broader understanding of the concept of excellence. It is recommended that further studies be performed using other study designs (eg grounded theory ${ }^{13}$ ) and the inclusion of multiple Iranian medical centers.

\section{Conclusion}

The findings of the present study provide a better understanding of the perception of the OR personnel on the concept of excellence, based on which optimal ethical care and an environment for the pursuit of excellence can be developed. The findings also provide evidencebased recommendations to nursing managers on how to gain the trust of patients and family caregivers, and promote the OR personnel's pursuit of personal, professional, organizational, and social excellence.

\section{Acknowledgments}

The present manuscript was extracted from a Ph.D. thesis. The authors would like to thank the participants for their time, effort, and contribution to the study.

\section{Disclosure}

The authors report no conflicts of interest in this work.

\section{References}

1. Santas G Socrates, Ellinika Grammata (in Hellenic) 1997.

2. Azgaly M. An Introduction to the Typology and Definition of Excellence Concept in Some Humanistic Schools. Journal of Research in Human Research Management. 2012;4(2):151-181.

3. Lucos A. Defining excellence in higher education. J High Educ. 2008;46.

4. Parham W. Toward clinical excellence in nursing. Nurse Educ Today J. 2002;7.

5. Abbasi M, Azar A, Mohebbi H, editors. Investigate the relationship between professional ethics and fundamental concepts of EFQM model in organizations' sustainable excellence. 6th International Conference on Economic Management and Engineering Sciences; 2016; Islamic Azad university: Sirjan Branch.

6. Dehghani M. EFQM Excellence model analysis using dynamic system. Manage Sci Iran. 2008;3.

7. Najmi M, Hoseini S. EFQM Excellence Model from Idea to Practice. Tehran: Saramad; 2009.

8. Stefaniak K. Discovering nursing excellence through appreciative inquiry. Nurse Leader. 2007;5(2):42-46. doi:10.1016/j.mnl.2007.01.010

9. Nikbakht A, Rezapour R. Nursing Professional Excellence Practical Model: A Qualitative Research Based on Payvar Theory. Scientific Research Journal of Rahbord. 2015;77:295-305.

10. Beeri I, Dayan R, Vigoda-Gadot E, Werner SB. Advancing ethics in public organizations: the impact of an ethics program on employees' perceptions and behaviors in a regional council. J Bus Ethics. 2013;112(1):59-78. doi:10.1007/s10551-012-1232-7

11. De Cremer D, Mayer DM, Schminke M. Guest editors' introduction: on understanding ethical behavior and decision making: A behavioral ethics approach. Bus Ethics Q. 2010;20(1):1-6. doi: $10.5840 /$ beq 20102012

12. Shleifer A. Does competition destroy ethical behavior? Am Econ Rev. 2004;94(2):414-418. doi:10.1257/0002828041301498

13. Ahmadi M. Survey of Workplace Violence and Related Factors in the Health Personnel in Operating Room in the Educational-Medical Centers of Esfahan University of Medical Sciences in 2017-18. Esfahan,Iran: Esfahan university of Medical Sciences; 2018.

14. Gilmartin J, Wright K. Day surgery: patients' felt abandoned during the preoperative wait. J Clin Nurs. 2008;17(18):2418-2425. doi:10.1111/j.1365-2702.2008.02374.x

15. Sanagoo A, Yazdani S, Jouybari L, Kalantari S. Uncivil behaviors in nursing workplace: a qualitative study. 2017.

16. Zadeh F, Lotfi M, Aqazadeh A, Asl Z. Viewpoints of operating room staff and students toward ethical climate in teaching hospitals of Tabriz University of Medical Sciences. Med Ethics J. 2015;9 (33):65-84.

17. Christian CK, Gustafson ML, Roth EM, et al. A prospective study of patient safety in the operating room. Surgery. 2006;139(2):159-173. doi:10.1016/j.surg.2005.07.037

18. Joudati A, Mohammad Bylankouhi E, Astanegi S. Compliance with universal precautions (ups) among operating room (or) personnel. Med J Tabriz Univ Med Sci. 2003;59:33-35.

19. Ticku MK, Mehta AK. Operating Room Management. Tehran: Jameenegar; 2011.

20. Mitchell L, Flin R, Yule S, Mitchell J, Coutts K, Youngson G. Evaluation of the scrub practitioners' list of intraoperative nontechnical skills system. Int J Nurs Stud. 2012;49(2):201-211. doi:10.1016/j.ijnurstu.2011.08.012

21. Schmock BN, Breckenridge DM, Benedict K. Effect of sacred space environment on surgical patient outcomes: a pilot study. Int J Human Caring. 2009;13(1):49-59. doi:10.20467/1091-5710.13.1.49 
22. Alfredsdottir H, Bjornsdottir K. Nursing and patient safety in the operating room. $J$ Adv Nurs. 2008;61(1):29-37. doi:10.1111/ jan.2008.61.issue-1

23. Sterchi LS. Perceptions that affect physician-nurse collaboration in the perioperative setting. AORN J. 2007;86(1):45-57. doi:10.1016/j. aorn.2007.06.009

24. Abolghasem GH, RAVAGHI H, PIROUZI M, MANSOURZADE A Utilizing Integrated Prospective and Retrospective Risk Analysis Method on General Processes Patient Flow in Operating Room in Seyed Alshohada Hospital in Semirom. Iran; 2013.

25. Farnia F, Abbaszadeh A, Borhani F. Barriers to developing the nurse-patient relationship in operation room: a qualitative content analysis. J Qual Res Health Sci. 2013;2(1):76-89.

26. Reynolds J, Carnwell R. The nurse-patient relationship in the post-anaesthetic care unit. Nurs Stand. 2009;24(15-17):40-46. doi:10.7748/ns2009.12.24.15.40.c7439

27. Graneheim UH, Lundman B. Qualitative content analysis in nursing research: concepts, procedures and measures to achieve trustworthiness. Nurse Educ Today. 2004;24(2):105-112. doi:10.1016/j.nedt.2003.10.001

28. Guba EG, Lincoln YS. Competing paradigms in qualitative research. Handbook Qual Res. 1994;2(163-194):105.

29. Lee Duckworth A, Steen TA, Seligman ME. Positive psychology in clinical practice. Annu Rev Clin Psychol. 2005;1:629-651. doi:10.1146/annurev.clinpsy.1.102803.144154

30. Kaviani M, Panagi A. Psychological Concepts in Quran. Ghom: Research Institute of Higher and University; 2014.

31. Winsent P. Amazing Positive Thinking Results. Tehran: Nandadesh Generation Publishing; 1998.

32. Farabi A. Thoughts of the Utopian Medina. Tehran: Ministry of Culture and Islamic Guidance/Printing and Publishing Organization; 2000.

33. Rezapour Nasrabad R. Leadership skills is necessary for extra roles behaviors and professional excellence. $Q J$ Nurs Manage. 2016;5 (1):9-16

34. Gallagher A. Moral distress and moral courage in everyday nursing practice. Online J Issues Nurs. 2011;16.

35. Murray JS. Moral courage in healthcare: acting ethically even in the presence of risk. Online J Issues Nurs. 2010;15(3).

36. Lachman VD, Murray JS, Iseminger K, Ganske KM. Doing the right thing: pathways to moral courage. Am Nurse Today. 2012;7(5):24-29.

37. Mohammadi S, Borhani F, Roshanzadeh M. Relationship between moral distress and moral courage in nurses. Iran J Med Ethics Hist Med. 2014;7(3).

38. Sadrollahi A, Khalili Z. Survey of professional moral sensitivity and associated factors among the nurses in West Golestan Province of Iran. Iran J Med Ethics Hist Med. 2015;8(3):50-61.

39. Moosavi S, Izadi A. Comparison of moral courage of the nurses and nursing managers working in hospitals affiliated to Shahid Beheshti University of Medical Sciences. Med Ethics J. 2017;11(41):17-24.

40. Walsh F. Strengthening Family Resilience. Guilford Publications; 2015

41. Rassool GH. The crescent and Islam: healing, nursing and the spiritual dimension. Some considerations towards an understanding of the Islamic perspectives on caring. J Adv Nurs. 2000;32(6):1476-1484. doi:10.1046/j.1365-2648.2000.01614.x

42. McBrien B. A concept analysis of spirituality. Br J Nurs. 2006;15 (1):42-45. doi:10.12968/bjon.2006.15.1.20309

43. Pesut B, Fowler M, Taylor EJ, Reimer-Kirkham S, Sawatzky R. Conceptualising spirituality and religion for healthcare. J Clin Nurs. 2008;17(21):2803-2810. doi:10.1111/j.1365-2702.2008.02344.x
44. Tanyi RA. Towards clarification of the meaning of spirituality. $J A d v$ Nurs. 2002;39(5):500-509. doi:10.1046/j.1365-2648.2002.02315.x

45. Razaghi N, Rafii F, Parvizy S, Sadat Hosseini A. Concept analysis of spirituality in nursing. Iran J Nurs. 2015;28(93):118-131. doi:10.29252/ijn.28.93.94.118

46. Homaunfar S. Excellency Pioneer, Introduction Excellence Organizations. Tehran: Human Resourse Study Publication; 2010.

47. Parsapoor A, Bagheri A, Larijani B. Patient's rights charter in Iran. Acta Med Iran. 2014;24-28.

48. Parsapoor A, Mohammad K, Malekafzali H, Aalaedini F, Larijani B. Regarding patients' right in Tehran University of Medical Sciences Hospitals. Ethics Med Hist. 2009;3(1):53-64.

49. Khademian Z, Vizeshfar F. Nursing students' perceptions of the importance of caring behaviors. J Adv Nurs. 2008;61(4):456-462. doi:10.1111/jan.2008.61.issue-4

50. Hull L, Sevdalis N. Teamwork and safety in surgery. Revista Colombiana De Anestesiología. 2015;43(1):3-6. doi:10.1016/j.rca.2014.10.002

51. O'Leary KJ, Buck R, Fligiel HM, et al. Structured interdisciplinary rounds in a medical teaching unit: improving patient safety. Arch Intern Med. 2011;171(7):678-684. doi:10.1001/archinternmed.2011.128

52. Zeltser MV, Nash DB. Approaching the evidence basis for aviation-derived teamwork training in medicine. Am J Med Qual. 2010;25(1):13-23. doi:10.1177/1062860609345664

53. Nguyen N, Watson WD, Dominguez E. An event-based approach to design a teamwork training scenario and assessment tool in surgery. J Surg Educ. 2016;73(2):197-207. doi:10.1016/j.jsurg.2015.10.005

54. Weller J. Assessing-teamwork-and-communication-in-the-healthprofessionsaspx; 2015. Available from: http://wwwsccmorg/ Communications/Critical-Connections/Archives/Pages/.

55. Baker VO, Cuzzola R, Knox C, et al. Teamwork education improves trauma team performance in undergraduate health professional students. J Educ Eval Health Prof. 2015;12:36. doi:10.3352/ jeehp.2015.12.36

56. Weaver SJ, Dy SM, Rosen MA. Team-training in healthcare: a narrative synthesis of the literature. BMJ Qual Saf. 2014;23 (5):359-372. doi:10.1136/bmjqs-2013-001848

57. Sonoda Y, Onozuka D, Hagihara A. Factors related to teamwork performance and stress of operating room nurses. J Nurs Manag. 2018;26(1):66-73. doi:10.1111/jonm.2018.26.issue-1

58. Hall P. Interprofessional teamwork: professional cultures as barriers. $J$ Interprof Care. 2005;19(Suppl 1):188-196. doi:10.1080/ 13561820500081745

59. Prati G, Pietrantoni L. Attitudes to teamwork and safety among Italian surgeons and operating room nurses. Work. 2014;49 (4):669-677. doi:10.3233/WOR-131702

60. Koppman P. Excellence: what is it? Whose Job is it? IRA, ARF cosponsored session, year book of the American Reading forum 2008.

61. Keshtkaran Z, Sharif F, Rambod M. Students' readiness for and perception of inter-professional learning: a cross-sectional study. Nurse Educ Today. 2014;34(6):991-998. doi:10.1016/j.nedt.2013.12.008

62. Moyer AR. Empowering patients, engaging teams: an interprofessional continuing education pilot. J Contin Educ Nurs. 2016;47 (9):421-426. doi:10.3928/00220124-20160817-09

63. Reader TW, Cuthbertson BH. Teamwork and team training in the ICU: where do the similarities with aviation end? Crit Care. 2011;15 (6):313. doi: $10.1186 / \mathrm{cc} 10353$

64. Lingard L, Regehr G, Espin S, Whyte S. A theory-based instrument to evaluate team communication in the operating room: balancing measurement authenticity and reliability. Qual Saf Health Care. 2006;15(6):422-426. doi:10.1136/qshc.2005.015388 


\section{Publish your work in this journal}

Risk Management and Healthcare Policy is an international, peerreviewed, open access journal focusing on all aspects of public health, policy, and preventative measures to promote good health and improve morbidity and mortality in the population. The journal welcomes submitted papers covering original research, basic science, clinical \& epidemiological studies, reviews and evaluations, guidelines, expert opinion and commentary, case reports and extended reports. The manuscript management system is completely online and includes a very quick and fair peer-review system, which is all easy to use. Visit http://www.dovepress.com/testimonials.php to read real quotes from published authors.

Submit your manuscript here: https:/www.dovepress.com/risk-management-and-healthcare-policy-journal 